\title{
REFERÊNCIAS BIBLIOGRÁFICAS PARA O ESTUDO CIENTÍFICO DA LEXICOGRAFIA E DA METALEXICOGRAFIA
}

Adja Balbino de Amorim Barbieri Durão Universidade Federal de Santa Catarina adjabalbino@gmail.com

Reinhold Werner

Universität Augsburg reinhold.werner@phil.uni-augsburg.de

\section{Manuais para a pesquisa sobre o dicionário}

ALVAR EZQUERRA, Manuel. "Lexicografía”. In:HOLTUS, MELTZELTIN, Michael Metzeltin; SCHMITT, Christian (orgs.). Lexikon der Romanistischen Linguistik. Tübingen: Max Niemeyer, 1992. p. 636-649.

BAJO PÉREZ, Elena. Los diccionarios. Introducción a la lexicografía. Gijón: Trea, 2000.

BÉJOINT, Henri; THOIRON, Philippe (eds.). Les dictionnaires bilingues. Louvain-la-Neuve: Duculot, 2000.

BÉJOINT, Henri. Modern Lexicography. An Introduction. Oxford - New York: Oxford University Press, 2000. 
BORBA, Francisco da Silva Organização de dicionários. Uma introdução à lexicografia. São Paulo: Editora UNESP, 2003.

COWIE, Anthony P. "Lexicography and its pedagogic applications. An introduction", Applied Linguistics, 2 , 3 (1981): 203-206.

ENGELBERG, Stefan; LEMNITZER, Lothar. Lexikographie und Wörterbuchbenutzung. Tübingen: Stauffenburg, 2001 (Stauffenburg-Einführungen 14).

GAUDIN, François; GUESPIN, Louis. Initiation à la lexicologie française. De la néologie aux dictionnaires. Bruxelles: De Boeck/ Duculot, 2000.

HARTMANN, Reinhard R. K. Teaching and Researching Lexicography. Harlow - München: Longman, 2001.

HAUSMANN, Franz Josef; REICHMANN, Oskar; WIEGAND, Herbert Ernst; ZGUSTA, Ladislav (eds.). Wörterbücher. Dictionaries. Dictionnaires. Ein internationales Handbuch zur Lexikographie. An International Encyclopedia of Lexicography. Encyclopédie internationale de lexicographie. Berlin - New York: de Gruyter, 1989-1991. (Handbücher zur Kommunikationswissenschaft 5.1, 5.2 e 5.3).

HERBST, Thomas; KLOTZ, Michael. Lexikografie. Paderborn München - Wien - Zürich: Schöningh 2003. (UTB 8263).

JACKSON, Howard. Lexicography: An introduction. London: Routledge, 2002.

LANDAU, Sydney I. Dictionaries. The Art and Craft of Lexicography. Cambridge: Cambridge University Press, 2001. 
MARCONI, Diego. Dizionari e enciclopedie. Torino: Giapichelli, 1986.

MARELLO, Carla. Dizionari bilingui. Con schede sui dizionari italiani per francese, inglese, spagnolo, tedesco. Bologna: Zanichelli, 1989 (Fenomeni linguistici 6).

MASSARIELLO MERZAGORA, Giovanna: La lessicografia. Bologna: Zanichelli, 1983.

MEDINA GUERRA, Antonia M. (coord.).Lexicografía española. Barcelona: Ariel, 2003.

PORTO DAPENA, Álvaro. Manual de técnica lexicográfica. Madrid: Arco, 2002.

RAFEL I FONTANALS, Joaquin. Lexicografia. Barcelona: VOX, 2005.

SCHLAEFER, Michael. Lexikologie und Lexikographie. Eine Einführung am Beispiel deutscher Wörterbücher. Berlin: Erich Schmidt, 2002 (Grundlagen der Germanistik 40). p. 74-200.

SVENSÉN, Bo. A Handbook of Lexicography. Cambridge: Cambridge University Press, 2009.

STERKENBURG, Piet van (ed.). A Practical Guide to Lexicography. Amsterdam -Philadelphia: John Benjamins, 2003 (Terminology and lexicography research and practice 6).

WELKER, Herbert Andreas. Dicionários. Uma pequena introdução à lexicografia. Brasília: Thesaurus, 2004. 
WIEGAND, Herbert Ernst. Wörterbuchforschung. Untersuchungen zur Wörterbuchbenutzung, zur Theorie, Geschichte, Kritik und Automatisierung der Lexikographie, 1. Teilband, Berlin New York: de Gruyter, 1998.

\section{Dicionários bibliográficos}

AHUMADA LARA, Ignacio (dir./ed.). Diccionario Bibliográfico de la Metalexicografía del Español (orígenes - año 2000), Jaén: Universidad de Jaén, 2006.

WIEGAND, Herbert Ernst. "Bibliographie zur Wörterbuchforschung von 1945 bis auf die Gegenwart. 2200 Titel. Ausgewählt aus germanistischer Perspektive“. In: WIEGAND, Herbert Ernst (ed.). Studien zur neuhochdeutschen Lexikographie VI, 2. Teilband, Hildesheim - Zürich - New York: Olms ), 1988 (Germanistische Linguistik 87 --90 1986). p. 627 - 821.

WIEGAND, Herbert Ernst. Internationale Bibliographie zur germanistischen Lexikographie und Wörterbuchforschung. Mit Berücksichtigung anglistischer, nordistischer, romanistischer, slavistischer und weiterer metalexikographischer Forschungen, Berlin - New York: de Gruyter, 2006 - 2008.

ZGUSTA, Ladislav. Lexicography Today. An annotated bibliography of the theory of lexicography. Tübingen: Max Niemeyer, 1988. (Lexicographica. Series Maior 18).

\section{Funções descritiva e prescritiva dos dicionários}

AZOFRA SIERRA, María Elena. "Criterio normativo y uso general culto en el Diccionario panhispánico de dudas". Revista de lexicografía XIII (2007): 7-16. 
COWIE, Anthony P. (ed.), The Dictionary and the Language Learner. Papers from the EURALEX Seminar at the University of Leeds, 1-3 April 198., Tübingen: Max Niemeyer, 1987 (Lexicographica. Series Maior, 17)

CUNDÍN SANTOS, Margarita.. "La norma lingüística del español y los conceptos coloquial y vulgar en los diccionarios de uso". Revista de lexicografía VIII (2001 - 2002): 43-102.

ETTINGER, Stefan (1982): "La variación lingüística en lexicografía”. In: HAENSCH, Günther, et al., La lexicografía. De la lingüística teórica a la lexicografía práctica. Madrid: Gredos, 1982 (Biblioteca Románica Hispánica III. Manuales, 56). p. 359-394.

GEERAERTS, Dirk. "Meaning and definition". In: STERKENBURG, Piet van (org.). A practical guide to lexicography. Amsterdam -- Philadelphia: John Benjamins, 2003. p. 83-93.

GLATIGNY, Michel (1989): "Les commentaires normatifs dans le dictionnaire monolingue". In: HAUSMANN, Franz Josef, et al. (eds.). Wörterbücher. Dictionaries. Dictionnaires. Ein internationales Handbuch zur Lexikographie. An International Encyclopedia of Lexicography. Encyclopédie internationale de lexicographie,), Teilband 1, Berlin - New York: de Gruyter, 1989 (Handbücher zur Kommunikationswissenschaft 5.1). p. 700-793.

LARA, Luis Fernando. "La cuestión de la norma en el Diccionario del español de México". In: Luis Fernando Lara. Dimensiones de la lexicografía. A propósito del Diccionario del español de México. México: El Colegio de México, 1990 (Jornadas 116). p. 157 $-193$.

LARA, Luis Fernando. Teoría del diccionario monolingüe., México: El Colegio de México, 1997 (Serie Estudios de Lingüística y Literatura XXXIII). p. 233-257. 
LUDWIG, Klaus-Dieter. " $\mathrm{Zu}$ normativen, konnotativen und stilistischen Angaben in Wörterbucheintragungen”. In: AGRICOLA, Erhard; SCHILDT, Joachim; VIEHWEGER Dieter (eds.). Wortschatzforschung heute. Aktuelle Probleme der Lexikologie und Lexikographie. Leipzig: VEB Verlag Enzyklopädie, 1982. p. $166-184$.

MALKIEL, Yakov (1989): "Wörterbücher und Normativität". In: HAUSMANN, Franz Josef, et al. (eds.). Wörterbücher. Dictionaries. Dictionnaires. Ein internationales Handbuch zur Lexikographie. An International Encyclopedia of Lexicography. Encyclopédie internationale de lexicographie, Teilband 1, Berlin - New York: de Gruyter 1989 (Handbücher zur Kommunikationswissen chaft 5.1). p. $63-69$.

RIPFEL, Martha (1989): "Die normative Wirkung deskriptiver Wörterbücher". In: HAUSMANN, Franz Josef et al. (eds.). Wörterbücher. Dictionaries. Dictionnaires. Ein internationales Handbuch zur Lexikographie. An International Encyclopedia of Lexicography. Encyclopédie internationale de lexicographie. Teilband 1, Berlin - New York: de Gruyter, 1989 (Handbücher zur Kommunikationswissenschaft 5.1). p. 189- 207.

\section{Distinção entre informação semântica e enciclopédica em dicionários}

ANAYA REVUELTA, Inmaculada. La definición enciclopédica. Estudio del léxico ictionímico. Madrid: Consejo Superior de Investiagaciones Científicas, 1999 (Biblioteca de Filología Hispánica 22).

ANAYA REVUELTA, Inmaculada. "Sobre el carácter enciclopédico de los diccionarios del español". Boletín de la Real Academia LXXX (2000): 177-207. 
BOSQUE, Ignacio. "Sobre la teoría de la definición lexicográfica”. Verba, v. 9 (1982): 105-123.

BOULANGER, Jean-Claude. Lexicographie générale, notes de cours. Québec: Université Laval/Société Dictionelinc, 1995.

CASTILLO CARBALLO, M. ${ }^{\text {a }}$; GARCÍA PLATERO, Juan Manuel. «La lexicografía didáctica». In: MEDINA GUERRA, Antonia M. (org.). Lexicografía Española. Barcelona: Ariel, 2003. p. 333-351.

ECO, Umberto. "Metaphor, Dictionary and Encyclopedia". New Literary History XV (1984): 255-271.

GUTIÉRREZ CUADRADO, Juan. "Enciclopedia y diccionario". In: FORGAS, Esther (ed.). Léxico y diccionarios. Tarragona: Universität Roviri i Virgili, 1996. p. 329 - 352

HAIMAN, John (1980): "Dictionaries and Encyclopedias". Lingua 50 (1980): 239-357.

LARA, Luis Fernando. «Dictionnaire de langue, enciclopédie et dictionnaire enciclopédique. Le sens de leur distinction?». In: HAUSMANN, Franz Josef, et al. (eds.), Wörterbücher. Dictionaries. Dictionnaires. Ein internationales Handbuch zur Lexikographie. An International Encyclopedia of Lexicography. Encyclopédie internationale de lexicographie. Teilband 1, Berlin-New York: de Gruyter , 1989 (Handbücher zur Kommunikationswissenschaft 5.1). p. 280-287.

PÜSCHEL, Ulrich (1981): "Bedeutungserklärungen als Regelund Sachbeschreibungen“. In: WIEGAND, Herbert Ernst (ed.). Studien zur neuhochdeutschen Lexikographie I, Hildesheim - New York, 1981(Germanistische Llinugistik 3-4/79). p. 123-138 
WERNER, Reinhold. "La definición lexicográfica". In: HAENS$\mathrm{CH}$, Günther et al. La lexicografía. De la lingüística teórica a la lexicografía práctica, Madrid: Gredos 1982 (Biblioteca Románica Hispánica III. Manuales, 56), p. 259-328; p. 269-272; p. 282- 285

WERNER, Reinhold (1983): "Semasiologische und enzyklopädische Definition im Wörterbuch". In: GÖTZ, Dieter Goetz; HERBST, Thomas (eds.). Theoretische und praktische Probleme der Lexikographie. 1. Augsburger Kolloquium. München: Hueber, 1983. p. 382-407.

WERNER, Reinhold. "Información terminológica, lingüística y enciclopédica", In: AHUMADA Lara, Ignacio (ed.). Lenguas de especialidad y lenguajes documentales. VII Jornada de la Asociación Española de Terminología Madrid, 24 de noviembre de 2006, Madrid: Asociación Española de Terminología, 2007. p. 3-18.

WIEGAND, Herbert Ernst (1988): "Was ist eigentlich Fachlexikographie? Mit Hinweisen zum Verständnis von sprachlichem und enzyklopädischem Wissen”. In: MUNSKE, Haider, et al. (eds.), Deutscher Wortschatz. Lexikologische Studien. Ludwig Erich Schmitt zum 80. Geburtstag von seinen Marburger Schülern. Berlin - New York: de Gruyter, 1988. p. 729-790.

WIEGAND, Herbert Ernst (1994): "Zur Unterscheidung von semantischen und enzyklopädischen Daten in Fachwörterbüchern". In: SCHAEDER, Burkhard; BERGENHOLTZ, Henning (eds.). Fachlexikographie. Fachwissen und seine Repräsentation in Wörterbüchern. Tübingen: Narr, 1994. p. 103-131.

\section{Sistemas conceituais e dicionários onomasiológicos}

REICHMANN, Oskar (1990): “Das onomasiologische Wörterbuch: Ein Überblick". In: HAUSMANN, Franz Josef Hausmann, et 
al. (eds.), Wörterbücher. Dictionaries. Dictionnaires. Ein internationales Handbuch zur Lexikographie. An International Encyclopedia of Lexicography. Encyclopédie internationale de lexicographie, Teilband 2, Berlin - New York: de Gruyter, 1990 (Handbücher zur Kommunikationswissenschaft 5.2). p. 1057-1066.

WOLF, Lothar. "Signo lingüístico y estructuras semánticas". In: HAENSCH, Günther, La lexicografía. De la lingüística teórica a la lexicografía práctica, Madrid: Gredos, 1982 (Biblioteca Románica Hispánica III. Manuales, 56). p. 329 - 358.

\section{O tratamento de processos de formação de palavrase o resultado em dicionários on-line e em dicionários bilíngues}

LÖTZSCH, Ronald. "Die Komposita im zweisprachigen Wörterbuch". In: HAUSMANN, Franz Josef, et al. (eds.). Wörterbücher. Dictionaries. Dictionnaires. Ein internationales Handbuch zur Lexikographie. An International Encyclopedia of Lexicography. Encyclopédie internationale de lexicographie, Teilband 3, Berlin - New York: de Gruyter, 1991 (Handbücher zur Kommunikationswissenschaft 5.3). p. $2779-2782$.

RETTIG, Wolfgang : "Die Wortbildungszusammenhänge im allgemeinen einsprachigen Wörterbuch", In: HAUSMANN, Franz Josef, et al. (eds.). Wörterbücher. Dictionaries. Dictionnaires. Ein internationales Handbuch zur Lexikographie. An International Encyclopedia of Lexicography. Encyclopédie internationale de lexicographie, Teilband 1, Berlin - New York: de Gruyter, 1989 (Handbücher zur Kommunikationswissenschaft 5.1).. p. 642-648. 


\section{Homonímia, polissemia e praxis da lematização lexicográ-} fica

ROTHE, Ulrike. Das einsprachige Wörterbuch in seinem soziokulturellen Kontext. Gesellschaftliche und sprachwissenschaftliche Aspekte in der Lexikographie des Englischen und des Französischen, Tübingen: Max Niemeyer, 1999 (Lexicographica. Series Maior 108). p. 114-174.

WERNER, Reinhold. "La definición lexicográfica". In: HAENSCH, Günther, et al., La lexicografía. De la lingüística teórica a la lexicografía práctica, Madrid: Gredos, 1982 (Biblioteca Románica Hispánica III. Manuales, 56). p. 259 -328.

\section{O tratamento de colocações em dicionários monolíngues e bilíngues}

BAHNS, Jens. Kollokationen als lexikographisches Problem. Eine Analyse allgemeiner und spezieller Lernerwörterbücher des Englischen. Tübingen: de Gruyter, 1996 (Lexicographica.Series Maior 74).

BUTINA-KOLLER, Ekaterina. "Le traitement des collocations dans les dictionnaires bilingues" In: DECESARIS, Janet; ALSINA, Victòria (orgs.): Estudis de Lexicografia 1999 - 2000. I Jornada de Lexicografia (3 de desembre de 1999). II Jornada de Lexicografia (24 de novembre de 2000. 2001. p. 129-136.

BUTINA-KOLLER, Ekaterina. Kollokationen im zweisprachigen Wörterbuch. Zur Behandlung lexikalischer Kollokationen in allgemeinsprachlichen Wörterbüchern des Sprachenpaares Französisch/ Russisch. Tübingen: Max Niemeyer 2005 (Lexicographica. Series Maior 124). 
BURGER, Harald. "Phraseologismen im allgemeinen einsprachigen Wörterbuch". In: HAUSMANN, Franz Josef, et al. (org.). Wörterbücher. Dictionaries. Dictionnaires. Ein internationales Handbuch zur Lexikographie. An International Encyclopedia of Lexicography. Encyclopédie internationale de lexicographie, Erster Teilband, Berlin - New York, 1989 (Handbücher zur Kommunikationswissenschaft 5.1). p. 593 - 599.

COP, Margaret. "Collocations in the bilingual dictionary". In: HAUSMANN, Franz Josef, et al. (orgs.). Wörterbücher. Dictionaries. Dictionnaires. Ein internationales Handbuch zur Lexikographie. An International Encyclopedia of Lexicography. Encyclopédie internationale de lexicographie, Dritter Teilband, Berlin - New York), 1991 (Handbücher zur Kommunikationswissenschaft 5.3). p. 2775-2778.

COWIE, Anthony P. "The place of illustrative material and collocations in the design of a learner's dictionary". In: STREVENS, Peter (ed.). In honour of A. S. Hornby Oxford: Oxford University Press, 1978. p. 127-139.

DOBROVOL'SKIJ, Dmitrij. "Zur lexikographischen Repräsentation der Phraseme (mit Schwerpunkt auf zweisprachigen Wörterbüchern)». In: MELLADO BLANCO, Carmen (ed.). Theorie und Praxis der idiomatischen Wörterbücher, Tübingen: Max Niemeyer, 2009 (Lexicographica. Series Maior 135). p. 149-168.

ETTINGER, Stefan. "Einige Probleme der lexikographischen Darstellung idiomatischer Einheiten (Französisch-Deutsch)". In: GRÉCIANO, Gertrud (ed.). Europhras 88. Phraséologie Contrastive. Actes du Colloque International Klingenthal - Strasbourg, 12-16 mai 1988. Strasbourg: Université des sciences humaines, départment d'études allemandes, 1989 (Collection Recherches germaniques (Strasbourg) 2). p. 95-116. 
MEL'ČUK, Igor. "Colocaciones en el diccionario". In: ALONSO RAMOS, Margarita (org.). Diccionarios y fraseología. A Coruña: Universidade da Coruña 2006 (Anexos de Revista de Lexicografía 3). p. 11-43.

MODEL, Benedikt. "Unidades fraseológicas en los diccionarios bilingües. Un problema de colocación". In: Diccionarios y fraseología, A Coruña: Universidade da Coruña, 2006 (Anexos de Revista de Lexicografía 3). p. 133-144.

PÖLL, Bernhard. Portugiesische Kollokationen im Wörterbuch: Ein Beitrag zur Lexikographie und Metalexikographie, Bonn: Romanistischer Verlag, 1996.

ROBERTS, Roda P. (1996): "Le traitement des collocations et des expressions idiomatiques dans les dictionnaires bilingues". In: BÉJOINT, Henri; THOIRON, Philippe (orgs.): Les dictionnaires bilingues. Louvain-la-Neuve: Duculot 1996. p. 181-197.

RUIZ MARTÍNEZ, Ana María. "La noción de colocación en las partes introductorias de algunos diccionarios monolingües del español”. Revista de Lexicografía XIII (2007): 139-182.

SCHEMANN, Hans. "Die Phraseologie im zweisprachigen Wörterbuch". In: HAUSMANN, Franz Josef, et al. (orgs.). Wörterbücher. Dictionaries. Dictionnaires. Ein internationales Handbuch zur Lexikographie. An International Encyclopedia of Lexicography. Encyclopédie internationale de lexicographie, Dritter Teilband, Berlin - New York, 1991 (Handbücher zur Kommunikationswissenschaft 5.3). p. 2789-2794.

STEINBÜGL, Birgit. Deutsch-englische Kollokationen. Erfassung in zweisprachigen Wörterbüchern und Grenzen der korpusbasierten Analyse, Tübingen: Max Niemeyer 2005 Lexicographica. Series Maior 126). 


\section{Pesquisas sobre o uso de dicionários}

ATKINS, Sue, (ed.). Using Dictionaries. Studies of Dictionary Use by Language Learners and Translators., Tübingen: Max Niemeyer, 1998 (Lexicographica. Series Maior 88)..

BÉJOINT, Henri. "The foreign student's use of Monolingual English Dicionaries: A study of language needs and reference skills". Applied Linguistcs, vol. II/3 (1981): 207-222.

BENSOUSSAN, Marsha; SIM, Donald; WEISS, Razelle. "The effect of dictionary use on EFL test performance compared with student and teacher attitudes and expectations". Reading in a Foreign Language, vol. 2/2 (1984): 262-276.

BOGAARDS, Paul. "Models of Dictionary Use». In: Nieuhuis, L.J.A. (ed.). Dutch Contributions to AILA ,93. 10th World Congress of the Association of Applied Linguistics. Selected in honour of Johan Matter. Amsterdam: Free University, 1993 p. 17-28.

WELKER, Herbert Andreas. O uso de dicionários: panorama geral das pesquisas empíricas. Brasília: Thesaurus, 2006.

\section{Problemas específicos da lexicografia monolíngue}

BUGUEÑO, Félix; FARIAS, Virgínia Sita. "Desenho da macroestrutura de um dicionário escolar de língua portuguesa". In: XATARA, Cláudia; BEVILACQUA, Claci Regina; HUMBLÉ, Philippe (orgs.). Lexicografia Pedagógica: Pesquisas e perspectivas. Florianópolis: UFSC/NUT, 2008. p. 129-167. Disponível em: $<$ http://www.cilp.ufsc.br/LEXICOPED.pdf $>$. 
BUGUEÑO, Félix. «Cómo leer y qué esperar de un diccionario monolingüe (con especial atención a los diccionarios del español)». Revista Língua \& Literatura, v. 8-9 (2003): 97-114.

BUGUEÑO, Félix. "Para uma taxonomia de paráfrases explanatórias". Alfa, v. 53/1 (2009): 243-260.

FARIAS, Virgínia Sita. "Dicionários escolares de língua portuguesa: uma breve análise de aspectos macroestruturais". Lusorama, v. 71/72 (2007): 160-206.

\section{Problemas específicos da lexicografia bilíngue}

BÉJOINT, Henri; THOIRON, Philippe. (orgs.). Les dictionnaires bilingues. Bruxelles: De Boeck/Duculot, 1996.

BENEDUZI, Renata; BUGUEÑO, Félix; FARIAS, Virginia. «Avanços na redação de um dicionário de falsos amigos espanhol-português». Lusorama, 61/62 (2005): 195-219.

CARVALHO, Orlene Lúcia de Sabóia. Lexicografia bilingüe português/alemão: Teoria e aplicação à categoria das preposições. Brasília: Thesaurus, 2001.

COWIE, Anthony. P. "The treatment of polisemy in the design of a learner's dictionary". In: HARTMANN, Reinhard R. K. (ed.). Dictionaries and their users. Proceedings of the 1978 BAAL Seminar on Lexicography. Exeter: University of Exeter, 1979 (Exeter Linguistic Series 4) p. 82-88.

DRYSDALE, Patrick. D. «The role of examples in a learner's dictionary». In: COWIE, Anthony P. (ed.), The Dictionary and the Language Learner. Papers from the EURALEX Seminar at the 
University of Leeds, 1-3 April 1985 (Lexicographica. Series Maior 17). Tübingen: Max Niemeyer, 1987. p. 213-223.

DURÃO, Adja Balbino de Amorim Barbieri. „Zur Verwendung zweisprachiger Wörterbücher als Hilfsmittel bei der Produktion fremdsprachlicher Texte“. Lebende Sprachen 58 (2013): p. 1-19.

DURÃO, Adja Balbino de Amorim Barbieri; SASTRE RUANO, María Ángeles; WERNER, Reinhold. „Equivalentes léxicos e informação semântica no Dicionário Contrastivo Português-Espanhol (DICOPOES)". In: DURÃO, Adja Balbino de Amorim Barbieri (org.). Por uma Lexicocgrafia Bilingue Contrastiva. Londrina: UEL, 2009. p. 193-207.

DURÃO, Adja Balbino de Amorim Barbieri; SASTRE RUANO, María Ángeles; WERNER, Reinhold. "Reflexiones en torno a un diccionario bilingüe contrastivo portugués-español". Boletim - Revista da Área de Humanas (Londrina) 53 (2011): 173-190.

DURÃO, Adja Balbino de Amorim Barbieri; SASTRE RUANO, María Ángeles; WERNER, Reinhold. "Lexicografía didáctica, diccionario bilingüe y lenguas afines: información semántica en el Diccionario contrastivo portugués-español (DiCoPoEs)". In: RULL, Antoni Nomdedeu; FORGAS BERDET, Esther; BARGALLÓ ESCRIVÀ, María (orgs.). Avances de lexicografía hispánica. Tarragona: Universitat Rovira i Virgili, 2012. p. 69-78.

DURÃO, Adja Balbino de Amorim Barbieri; WERNER, Reinhold. "Caminhos para a seleção da nominata de um dicionário de falsos amigos". Working Papers em Linguística 12 - número especial (2011): 43-53.

WERNER, Reinhold. "Diccionarios bilingües del español y otra lengua iberorrománica: elementos de una teoría lexicográfica para 
combinaciones específicas de lenguas". In: CASTILLO CARBALLO, M. ${ }^{a}$ Auxiliadora; GARCÍA PLATERO, Juan Manuel (orgs.): La lexicografía en su dimensión teórica, Málaga: Servicio de Publicaciones e Intercambioi Científico de la Universidad de Málaga, 2010 (Estudios y Ensayos 125). p. 643-656.

ZÖFGEN, Ekkehard. "Bilingual Learner's Dictionaries". In: HAUSMANN, Franz Josef, et al. (Hrsg.): Wörterbücher. Dictionaries. Dictionnaires. Ein internationales Handbuch zur Lexikographie. An International Encyclopedia of Lexicography. Enciclopédie internationale de lexicographie, Dritter Teilband. Berlin - New York, 1991 (Handbücher zur Kommunikationswissenschaft 5.3). p. 2888-2903.

ZÖFGEN, Ekkehard. Lernerwörterbücher in Theorie und Praxis. Ein Beitrag zur Metalexikographie mit besonderer Berücksichtigung des Französischen. Tübingen: Max Niemeyer, 1994 (Lexicographica. Series Maior 59).

\section{Elementos para a avaliação de dicionários}

FUENTES MORÁN, M. ${ }^{a}$ Teresa; GARCÍA PALACIOS, José Joaquín; TORRES DEL REY, Jesús. "Algunos apuntes sobre la evaluación de diccionarios". Revista de Lexicografía XI (20042205): 69-80.

SALVADOR, Gregorio. "La crítica de diccionarios". In: ÁLVAREZ DE MIRANDA, Pedro; POLO, José (orgs.). Lengua y diccionarios. Estudios ofrecidos a Manuel Seco. Madrid, 2002. p. 235-239.

STEINER, Roger. "Guidelines for Reviews of Bilingual Dictionaries”. Dictionaries 6 (1984): 166-181. 
WERNER, Reinhold. „Ein vergleichender Test dreier Wörterbücher Spanisch-Deutsch/Deutsch-Spanisch“. Hispanorama 47 (1987): 159-171, 49 (1988): 152-167, 50 (1988): 167-175.

WIEGAND, Herbert Ernst (ed.). "Wörterbuchkritik. Dictionary Crticism”. Lexicographica 9 (1994): 1-173.

\section{Lematização, Macro e Microestrutura em dicionários bilíngues}

BERGENHOLTZ, Henning; TARP, Sven; WIEGAND, Herbert Ernst (1999): "Datendistributionsstrukturen, Makro- und Mikrostrukturen in neueren Fachwörterbüchern". In: HOFFMANN, Lothar; KALVERKÄMPER, Hartwig; WIEGAND, Herbert (orgs.). Fachsprachen. Languages for Special Purposes. Ein internationales Handbuch zur Fachsprachenforschung und Terminologiewissenschaft. An International Handbook of Special-Language and Terminology Research, 2. Halbband, Berlin - New York 1999 (Handbücher zur Sprach-und Kommunikationswissenschft 14.2). p. $1762-1832$.

SCHNORR, Veronika. "Problems of Lemmatization in the Bilingual Dictionary". In: HAUSMANN, Franz Josef, et al. (orgs.). Wörterbücher. Dictionaries. Dictionnaires. Ein internationales Handbuch zur Lexikographie. An International Encyclopedia of Lexicography. Encyclopédie internationale de lexicographie, Dritter Teilband, Berlin - New York, 1991 (Handbücher zur Kommunikationswissenschaft 5.3). p. 2813-2817.

WERNER, Reinhold. "La selección de lemas en los diccionarios español-alemán y alemán-español o ¿un diccionario de qué lengua es un diccionario de las lenguas española y alemana?". In: FUENTES MORÁN, Ma Teresa; WERNER, Reinhold (orgs.) Lexicogra- 
fias iberorrománicas: problemas, propuestas y proyectos. Madrid - Frankfurt am Main, 1998. p. 139 - 158.

WIEGAND, Herbert Ernst. (1983): "Was ist eigentlich ein Lemma? Ein Beitrag zur Theorie der lexikographischen Sprachbeherrschung". In: Herbert Ernst Wiegand (Hrsg.): Studien zur neuhochdeutschen Lexikographie III, Zürich - New York - Hildesheim, 1983. p. $401-474$.

WIEGAND, Herbert Ernst (1989). "Aspekte der Makrostruktur im allgemeinen einsprachigen Wörterbuch: Alphabetische Anordnungsformen und ihre Probleme". In: Franz Josef Hausmann et al. (org.): Wörterbücher. Dictionaries. Dictionnaires. Ein internationales Handbuch zur Lexikographie. An International Encyclopedia of Lexicography. Encyclopédie internationale de lexicographie, Erster Teilband, Berlin - New York, 1989. p. 409 - 462.

WIEGAND, Herbert Ernst (1998): "Altes und Neues zur Makrostruktur alphabetischer Printwörterbücher". in: Herbert Ernst Wiegand (Hrsg.): Wörterbücher in der Diskussion III. Vorträge aus dem Heidelberger Lexikographischen Kolloquium III, Tübingen, 1998. p. $348-372$.

\section{Informação sobre a morfologia flexional em dicionários bilíngues}

FUENTES MORÁN, María Teresa. Gramática en la lexicografía bilingüe. Morfología y sintaxis en diccionarios español-alemán desde el punto de vista del germanohablante, Tübingen, 1997.

MUGDAN, Joachim. "Information on Inflectional Morphology in the General Monolingual Dictionary". In: Franz Josef Hausmann et al. (orgs.): Wörterbücher. Dictionaries. Dictionnaires. 
Ein internationales Handbuch zur Lexikographie. An International Encyclopedia of Lexicography. Encyclopédie internationale de lexicographie, Erster Teilband, Berlin - New York, 1989. p. 518 -525 .

CALUWE, Johan de; TAELDEMAN, Johan (2003): "Morphology in dictionaries". In: Piet van Sterkenburg (orgs.): A Practical Guide to Lexicography". Amsterdam - Philadelphia, 2003. p. 114 -126 .

ETTINGER, Stefan. "Die Modifikation in der Lexikographie". In: Dieter Götz; Thomas Herbst (orgs.): Theoretische und praktische Probleme der Lexikographie. 1. Augsburger Kolloquium, München, 1984. p. 63 - 106.

ETTINGER, Stefan. „Um dicionário alemão-português de verbos com prefijos". In: $\mathrm{M}^{\mathrm{a}}$ Teresa Fuentes Morán; Reinhold Werner (orgs.): Lexicografías iberorrománicas: problemas, propuestas y proyectos, Madrid - Frankfurt am Main, 1998. p. 185 - 199.

LÖTZSCH, Ronald. "Die Komposita im zweisprachigen Wörterbuch". In: Franz Josef Hausmann et al. (orgs.): Wörterbücher. Dictionaries. Dictionnaires. Ein internationales Handbuch zur Lexikographie. An International Encyclopedia of Lexicography. Encyclopédie internationale de lexicographie, Dritter Teilband, Berlin - New York, 1991. p. 2779 - 2783.

RETTIG, Wolfgang. "Die Wortbildungszusammenhänge im allgemeinen einsprachigen Wörterbuch". In: Franz Josef Hausmann et al. (orgs.): Wörterbücher. Dictionaries. Dictionnaires. Ein internationales Handbuch zur Lexikographie. An International Encyclopedia of Lexicography. Encyclopédie internationale de lexicographie, Erster Teilband, Berlin - New York, 1989. p. 642 - 649. 


\section{Informação sobre valência verbal em dicionários bilíngues}

COWIE, Anthony Paul. "Information on Syntactic Constructions in the General Monolingual Dictionary". In: Franz Josef Hausmann et al. (orgs.): Wörterbücher. Dictionaries. Dictionnaires. Ein internationales Handbuch zur Lexikographie. An International Encyclopedia of Lexicography. Encyclopédie internationale de lexicographie, Erster Teilband, Berlin - New York, 1989. p. 588 -592 .

FUENTES MORÁN, María Teresa. Gramática en la lexicografía bilingüe. Morfología y sintaxis en diccionarios español-alemán desde el punto de vista del germanohablante, Tübingen, 1997.

KROMANN, Hans-Peder; RIIBER, Theis; ROSBACH, Poul. "Grammatical Constructions in the Bilingual Dictionary", in: Franz Josef Hausmann et al. (org.). Wörterbücher. Dictionaries. Dictionnaires. Ein internationales Handbuch zur Lexikographie. An International Encyclopedia of Lexicography. Encyclopédie internationale de lexicographie, Dritter Teilband, Berlin - New York, 1991. p. 2770 - 2775.

\section{A equivalencia no dicionário bilíngue}

DUVAL, Alain (1991): "L'équivalence dans le dictionnaire bilingue". In: Franz Josef Hausmann et al. (org.): Wörterbücher. Dictionaries. Dictionnaires. Ein internationales Handbuch zur Lexikographie. An International Encyclopedia of Lexicography. Encyclopédie internationale de lexicographie, Dritter Teilband, Berlin - New York, 1991. p. 2817 - 2824.

SZENDE, Thomas. "Problèmes d'équivalence dans les dictionnaires bilingues", in: Henri Béjoint / Philippe Thoiron (Hrsg.): Les dictionnaires bilingues, Louvain-la-Neuve, 1996, p. 111 - 126. 
WERNER, Reinhold. Rezensionsaufsatz zu Rudolf J. Slabý; Rudolf Grossmann; Carlos Illig. Wörterbuch der spanischen und deutschen Sprache, I: Spanisch-Deutsch, Wiesbaden. Iberoamericana 63/64 ([1994] 1996): 85 - 99.

WERNER, Reinhold. "Das Problem der Äquivalenz im zwei- und im mehrsprachigen Fachwörterbuch”. In: Lothar Hoffmann; Hartwig Kalverkämper; Herbert Ernst Wiegand (orgs.): Fachsprachen. Languages for Special Purposes. Ein internationales Handbuch zur Fachsprachenforschung und Terminologiewissenschaft. An International Handbook of Special-Language and Terminology Research, 2. Halbband, Berlin - New York, 1999. p. 1853 - 1884.

WERNER, Reinhold. "Äquivalenz im zweisprachigen Wörterbuch". In: Chabarovskij Gosudarstvennyj Pedagogičeskij Universitet (orgs.): Vostok-Zapad: Naučnoe sotrudničestvo po germanskoj $i$ romanskoj filologii. Sbornik naučnych trudov po materialam meždunarodnoj konferencii < <Zapad - Vostok. Obrazovanie I nauka na poroge XXI veka > > . Germanistik und Romanistik: Wissenschaft zwischen Ost und West. Materialien der internationalen wissenschaftichen Konferenz $<<$ West-Ost: Bildung und Wissenschaft an der Schwelle des 21. Jahrhunderts > >, Chabarovsk, 2002. p. $53-76$.

WERNER, Reinhold. "El problema de la equivalencia en los diccionarios bilingües especializados". In: María Teresa Fuentes Morán / Reinhold Werner (Hrsg.): Diccionarios: textos con pasado y futuro, Frankfurt am Main - Madrid, 2002. p. 3 - 20.

WIEGAND, Herbert Ernst (2002): “Äquivalenz in zweisprachigen Printwörterbüchern. Kritik und Vorschläge”, in: Herbert Ernst Wiegand (Hrsg.): Studien zur zweisprachigen Lexikographie mit Deutsch VIII, Hildesheim - Zürich - New York, 2002. p. 112 - 175. 
WIEGAND, Herbert Ernst (2002): "Equivalence in Bilingual Lexicography: Criticism and Suggestions". Lexikos 12, 2002. p. 239 -255 .

WIEGAND, Herbert Ernst. "On the Lexicographical Description of Equivalent Open Class Expressions". Lexikos 13, 2003. p. 38 -64 .

WIEGAND, Herbert Ernst. “Äquivalentpräsentation und Wörterbuchfunktion in zweisprachigen Printwörterbüchern. Mit einem Seitenblick auf die so genannte moderne lexikographische Funktionenlehre", in:Birgit Igla / Pavel Petkov / Herbert Ernst Wiegand (Hrsg.): Kontrastive Lexikologie und zweisprachige Lexikographie. 2. Internationales Kolloquium zur Wörterbuchforschung St. Kliment Ohridski-Universität Sofia 18. bis 19. Oktober 2002, Hildesheim - Zürich - New York, 2005. p. 1 - 38.

WIEGAND, Herbert Ernst. “Äquivalenz, Äquivalentdifferenzierung und Äquivalentpräsentation in zweisprachigen Wörterbüchern. Eine neue einheitliche Konzeption”. In: Henrik Gottlieb; Jens Erik Morgensen; Arne Zettersten (orgs.): Symposium on Lexicography XI. Proceedings of the Eleventh International Symposium on Lexicography May 2-4, 2002, at the University of Copenhagen, 2005. p. $17-57$.

WIEGAND, Herbert Ernst. "Zur lexikografischen Beschreibung nennlexikalischer äquivalenter Wortschatzeinheiten". In: Doris Steffens (orgs.): Wortschatzeinheiten. Aspekte ihrer (Be)schreibung: Dieter Herberg zum 65. Geburtstag, Mannheim, 2005. p. $43-75$. 


\section{Informação intercultural em dicionários bilíngues}

BERNOT, Denise. "La lexicographie bilingue: un chemin semé d'embuches d'une culture à une autre”. In: Thomas Szende (Hrsg.): Dictionnaires bilingues. Méthodes et contenus, Paris, 2000. p. 53 -60 .

NGUYEN, Dinh-Hoa. "Bicultural Information in a Bilingual Dictionary". In: Ladislav Zgusta (Hrsg.): Theory and Method in Lexicography: Western and Non-Western Perspectives, Columbia, S.C., 1980. p. $163-175$.

NGUYEN, Dinh-Hoa. "Teaching Culture through bilingual dictionaries”. Dictionaries 2 - 3, 1980. p. 57 - 68 .

REY, Alain. "Les écarts culturels dans les dictionnaires biingues", in: Lexicographica 2, 1986. p. $33-42$.

REY, Alain. «Divergences culturelles et dictionnaire bilingue“. In: Franz Josef Hausmann et al. (org.). Wörterbücher. Dictionaries. Dictionnaires. Ein internationales Handbuch zur Lexikographie. An International Encyclopedia of Lexicography. Encyclopédie internationale de lexicographie, Dritter Teilband, Berlin - New York, 1991. p. $2865-2870$.

TOMASZCZYK, Jerzy. «The culture-bound element in bilingual dictionaries». In: Reinhard R. K. Hartmann (org.): LEXeter '83 Proceedings. Papers from the International Conference on Lexicography at Exeter, 9 - 12 September, 1983, Tübingen, 1984. p. $289-297$. 